\title{
Dietary Partitioning in Newly Sympatric Urban Flying-foxes (Pteropus poliocephalus and Pteropus alecto)
}

\author{
Phoebe Griffith ${ }^{* 1,2}$, Kerryn Parry-Jones ${ }^{3}$ and Andrew A. Cunningham ${ }^{1}$ \\ ${ }^{1}$ Institute of Zoology, Zoological Society of London, Regent's Park, London, UK. NW1 4RY \\ ${ }^{2}$ Royal Veterinary College, University of London, Royal College Street, London, UK. NW1 0TU \\ ${ }^{3}$ School of Life and Environmental Sciences, University of Sydney, Sydney 2006, NSW Australia \\ *Corresponding author: phoebe.griffith@zoo.ox.ac.uk, +44 7876643834
}

Key Words: > fruit bat $>$ global change $>$ range expansion $>$ resource competition 


\begin{abstract}
The black flying-fox (Pteropus alecto) is extending its range southward and is now sympatric with the grey-headed flying-fox, (P. poliocephalus) in New South Wales. Competition for food between the two species has been suggested as a contributor to declines of the vulnerable grey-headed flying-fox. During winter 2016 the diet of both species was investigated over one night at four sites of sympatry, by microscopic analysis of faecal samples. Resource partitioning between the two species was found, with the black flying-fox either preferentially choosing to eat more fruit than the grey-headed flying-fox or being an inferior competitor for pollen and nectar. These results, though limited, do not support the hypothesis that the black flying-fox threatens the grey-headed flying-fox through food competition.
\end{abstract}

\title{
Introduction
}

Increasing human impact on the global environment is causing unprecedented changes in terrestrial ecosystems. Wildlife species can respond to these changes by altering geographic distribution ('range shifts') (Ancillotto et al. 2016). However, these responses can have knock on effects such as altering inter-specific interactions (Alexander et al. 2015).

Range shift is exemplified by Pteropus bats (flying-foxes) in Australia; in particular the black flying-fox (Pteropus alecto) and the grey-headed flying-fox (Pteropus poliocephalus).

Historically (pre-1920s), the southern limit of the distribution of the black flying-fox was $23^{\circ} \mathrm{S}$, corresponding with the northern limit of the grey-headed flying-fox. As the range of the black flying-fox has extended southwards during the last century the two species increasingly occur in sympatry on the eastern coast of Australia (Roberts et al. 2012). Although the range of the grey-headed flying fox has remained constant, its abundance has declined in areas of species overlap (Roberts et al. 2012). It has been repeatedly suggested that the range expansion of the black flying-fox may threaten the grey-headed flying-fox through competition for food (Eby et al. 1999; Birt and Markus 1999; Welbergen 2004), possibly contributing to the decline of grey-headed flying-fox and its assessment as vulnerable by the IUCN.

The diet of flying-foxes is hypothesized to be a driver of range shifts (McWilliam 1986) and 
resultant inter-specific competition (Eby et al. 1999). Both black and grey-headed flyingfoxes are dietary generalists, feeding on a wide range of plant matter. Grey-headed flyingfoxes are sequential specialists (feeding on foods in a hierarchy of preference) (Parry-Jones and Augee 1991, 2001). Nectar and pollen of native trees, particularly those in the families Myrtaceae and Proteaceae, are the preferred food source, and Ficus spp. are also important (Schmelitschek et al. 2009.) Black flying-fox diet preferences are less well documented. This species will eat a range of native and exotic plant matter, tracking locally abundant food sources (Palmer et al. 2000). Both species are highly mobile (Webb and Tidemann 1996). It has been suggested that the diet of each species is equivalent where their ranges overlap (Hall and Richards 2000).

Here, we investigate the degree of competition between these two newly sympatric species of flying-fox by microscopically identifying dietary items from faeces collected at four urban sites of sympatry. If black flying-foxes compete with grey-headed flying-foxes for food resources, we hypothesise that both species would be eating similar foods at these sites.

\section{Materials and Methods}

\section{Study Sites and Faecal Sample Collection}

Four study sites were used in north-eastern New South Wales, Australia, during early June 2016 (Table 1). All sites are forest fragments in urban parks which have been used as daytime roosts by both flying-fox species since at least 2010 (Parry-Jones pers.com).

Diet was investigated by collection of faeces at each site. Before dusk, 10 plastic sheets (3.6 $\mathrm{m} \times 2.6 \mathrm{~m}$ ) were placed beneath each flying-fox roost. The sheets were scattered broadly across the site, with placement ensuring faeces were collected from both species once they returned from feeding. The following morning 10 faecal samples were collected from each sheet, with individual samples defined as a single isolated dropping. This was done once per site with each sample being placed in a separate, sealed plastic bag, and frozen for later analysis. Since flying-fox gut-passage time is very short (Tedman and Hall 1985) faecal samples will represent food eaten during the collection night. 
Immediately before collection, observations were recorded on the number and species composition of flying-foxes roosting within the sampling range of each sheet, and used to determine the probability that a given faecal sample was from a black or grey-headed flying-fox. Therefore, each faecal sample on a sheet with a mixed aggregation hanging above had a probability of being from either species, rather than a particular sample being directly attributable to a species. The number of Pteropus individuals occupying each site was estimated by counting flying-foxes at evening 'fly-out' (as in Parry-Jones and Augee 1992a) on the evening the sheets were placed at the site. The percentage of each species of flying-fox using a site was estimated from ground observations along transects criss-crossing each roost.

\section{Diet Analysis}

Items present in each faecal sample were identified using a modified version of the method described by Parry-Jones and Augee (2001). This method minimally alters the faecal sample contents, often composed of the soft parts of fruit which could be rendered unidentifiable by straining or drying.

Each faecal sample was thawed and three sub-samples of approximately $4 \mathrm{~mm}^{3}$ were mounted onto microscope slides. A drop of water was added to one sub-sample to use actual colour for identification. The remaining two sub-samples were each stained with a drop of $1 \%$ aqueous safranin solution (Jones 2012). Three sub-samples have been shown to be representative of the entire faecal sample in these flying-fox species (López-Baucells, unpublished data). Each slide was examined by light microscopy at x100 and x400 magnification by scanning side-to-side transects over the entire slide. Plant matter was identified using a reference collection (ParryJones, unpublished) and published references (APSA Members, 2007; Shimeld et al. 2000). Unidentifiable plant materials were recorded as ‘unknown' morphospecies.

Flying-foxes are capable of removing the protein from within the resistant exine exteriors of certain pollen grains (Parry-Jones 1993), so can digest pollen grains as a dietary component. It is not possible to detect nectar through faecal microscopy; nectar consumption can only be inferred by pollen occurrence and corroborated by published behavioural observations. In this study pollen occurrence was recorded as either undigested or digested. Intact pollens that occurred rarely and were from plants lacking floral nectaries were considered contaminants e.g. acacia pollen. 
All plant materials in the diet were identified to the lowest taxon level possible. It was not possible to distinguish between most species of Myrtaceae pollen grains. Pollens grains with a polar size of $<20$ micrometres were identified as Melaleuca spp. and those $>20$ micrometres as Eucalyptus or Corymbia spp. (López-Baucells, pers. com.).

\section{Statistical Analysis}

Differences in diet composition between the flying-fox species were analysed by comparing a matrix of Bray-Curtis dissimilarity in diet composition (Baldoa and Drake 2002) between sheets to a matrix of Euclidean distance dissimilarity in flying-fox species composition using a Mantel test. Flying-fox species composition for each sheet was given a value between 0 (all P. poliocephalus) and 1 (all P. alecto). A significant result (correlation coefficient (r) of more than 0.3; Hinkle et al. 2003) indicates an association between diet composition in faecal samples and species composition of flying-fox. P-values were generated by 10,000 permutations of one of the matrices.

Difference between flying-fox species in food group was investigated by comparing associations between occurrence of dietary groups (fruit, pollen or introduced plant) in faeces and probability that a given sample was from a black flying-fox, using a Generalised Linear Mixed-Effect Model (GLMM) with binomial error structure and a logit link function for each food group. Food group presence/absence was the dependent variable and probability the sample was from a black flyingfox was the continuous predictor, with site included as a random effect to account for betweensite variability. Significant values $(\mathrm{P}=0.01)$ showed an association between species and occurrence of a given dietary item. Unknown fruits and pollens were excluded from the GLMMs.

The relative frequency of occurrence of a given diet item was expressed in comparison to the occurrence of all other identified items in a given set of samples (Parry-Jones and Augee 1991). Statistical analyses were carried out using R (R Core Team 2013), in the \{vegan\} (Oksanen et al. 2015), \{glmm\} (Knudson 2018), or \{stats\} (R Core Team 2013) package.

\section{Results}

\section{Colony Composition}


The approximate colony size in Lismore was 65,000; Nambucca 20,000; Port Macquarie 100,000 and Wingham >100,000. The percent composition of black flying-foxes to greyheaded flying-foxes was 50:50 in Lismore and Wingham, 20:80 in Nambucca and 5:95 in Port Macquarie.

\section{Diet Composition}

Our sampling produced 386 faecal samples with 100 samples from three sites and 86 collected from Lismore. A summary of the results is given in Table 2. Myrtaceae pollen was the most frequently occurring dietary item, comprising $51 \%$ of identifiable plant material. Banksia spp. pollen, Avicennia marina leaf and the fruits of Ficus spp., Melia azedarach, Ligustrum lucidium and Solanum mauritanium frequently occurred in faecal samples. Macadamia spp. pollen occurred regularly, but was undigested. All other identified items occurred at $<1 \%$ frequency. Native species predominated over introduced or cultivated species, with a relative frequency of occurrence of $84 \%$. Twenty faecal samples also contained the remains of large insects.

\section{Dietary Differences Between Flying-Fox Species}

A Mantel correlation analysis showed a significant, positive correlation between dissimilarity matrices of diet composition and flying-fox species $(\mathrm{r}=0.4186, \mathrm{p}<0.001)$. Diet composition, therefore, differed between flying-fox species with individuals within a species having more similar diet composition within a species than between species.

Occurrence of fruit in faecal samples increased with increasing probability that a faecal sample was from a black flying-fox (GLMM: Odds Ratio = 7.29; CI:3.26-16.27; p=0.01), (Fig. 1 a). Occurrence of pollen in faecal samples decreased with increasing probability that a faecal sample was from a black flying-fox (GLMM: OR:0.38; CI:0.20-0.73; p=0.01), (Fig. $1 b$ ). Occurrence of introduced plants in faecal samples on a sheet increased with increasing probability that a faecal sample was from a black-flying fox (GLMM: OR:2.51; CI:1.38-4.58; p=0.01), (Fig. $1 c$ ). The relative frequency of occurrence of fruit and pollen reflects the estimated proportions of each of the two species roosting at each site with more fruit consumed at sites with higher overall proportions of black flying-foxes (Fig. 2).

\section{Discussion}


The sites in this study had medium to very large colonies of flying-foxes in residence. Colony size is related to the available food supply and large winter colonies are associated with availability of flowering forest eucalypts (Parry-Jones 1985) so it is likely that this study occurred when this preferred food source was abundant. The dietary items found in the present study are consistent with the range of dietary items known to be consumed by greyheaded and black flying-foxes.

Myrtaceae pollen was the most frequently consumed dietary item. This supports a reported preference for such pollen when available, with flying-foxes known to concurrently drink the nectar (Parry-Jones and Augee 1991, 2001). Banskia pollen was also an important dietary item. Both Myrtaceae and Banksia pollen grains were digested, and it is likely that both nectar and pollen were consumed from these blossoms. Macadamia pollen also occurred frequently in faeces, this is likely for nectar as Macadamia pollen grains did not show evidence of digestion: this requires confirmation by observation of flying-fox feeding behaviour, especially due to the small size of the flowers of Macadamia inflorescences. However, bats will eat nectar from small flowers if they occur in inflorescences e.g. Banksia. All identified pollen was native to north-eastern New South Wales, although Macadamia trees predominantly occur in cultivation. A range of native and introduced fruits was also consumed.

Leaves of Avicennia marina were widely consumed, possibly for their salt content (ParryJones and Augee 1991). Large arthropod parts present in the faecal samples are likely due to deliberate insectivory. There is a growing body of evidence suggesting insectivory is common in Pteropodids (Parry-Jones and Augee 1992b, Barclay et al. 2006) possibly to supplement protein intake and to meet nitrogen requirements.

Fruit remains were significantly more likely to be found in faecal samples with a higher probability of being from black flying-foxes than from grey-headed flying-foxes, with the latter more likely to contain pollen/nectar. These findings show that grey-headed flyingfoxes are able to consume their preferred food sources even when in sympatry with black flying-foxes, and support suggestions that black flying-foxes are more frugivorous than greyheaded flying-foxes (Nelson 1965; McWilliam 1986; Birt 2004). 
This study reflects diet over just one night at each site, and the results cannot be extrapolated to different seasons or different circumstances of resource availability. In particular, during resource scarcity the ability of the two-species to compete for limited food items may differ. When grey-headed flying-foxes have to rely on less-preferred food items, such as fruit, competition may increase. Our results also contain a relatively small number of samples with a high probability of being from black flying-foxes, so species differences may be less distinct in our results.

When morphologically and ecologically similar species occur in sympatry, they do so by partitioning limiting resources, such as food, to avoid competitive exclusion (Hardin 1960). Since the hierarchy of food preference for grey-headed flying-foxes is well established, (with pollen/nectar of the Myrtaceae and Proteaceae as the 'first choice'), the greater occurrence of fruit we found in the black flying-fox diet is either due to an intrinsic preference, or because black flying-foxes are outcompeted by grey-headed flying-foxes for floral resources and so consumes fruit as an available second option. However, competitive exclusion theory applies to stable environments (Chesson 2000) and given the heterogeneity of Pteropus food availability in space and time, the presence or degree of competition may fluctuate as resource provision fluctuates. In mixed-species roosts occupancy patterns differ between Pteropus spp. with grey-headed flying fox population size showing more extreme fluctuation than that of the black flying-fox (Birt 2004; P. Eby, pers. obs.). This hints at a reliance on different food resources, as these are a key factor in Pteropus movements (Eby 1996; Palmer and Woinarski 1999), as would be expected if there was a partitioning of food resources.

The reasons for the southward expansion of black flying-foxes are unknown, with Roberts $e t$ al. (2012) rejecting climate change and conversion of native forest to agricultural and urban land as an explanation. Two alternative mechanisms should be considered. Firstly, if greyheaded flying-foxes are the superior competitor, the southward expansion of black flyingfoxes may be a response, rather than a contributing factor, to grey-headed flying-fox declines (Roberts et al. 2012). Loss of habitat that provides flowering resources would be more likely to cause population declines in the more nectarivorous grey-headed flying-fox. Secondly, the current study shows that black flying-foxes consume introduced plants more often than greyheaded flying foxes. If black flying-foxes are more frugivorous, then the southward expansion may be to exploit fruit resources, including recently-naturalised plants such as wild tobacco and privet, which fruit in the resource-poor winter. 
This study provides evidence that when grey-headed and black flying-foxes occur in sympatry there is partitioning of food resources. The results do not support the view that the expansion of the black flying-fox is a contributing factor to the decline of the grey-headed flying-fox due to food competition. Black flying-foxes are not outcompeting grey-headed flying-foxes if the latter can forage on the more favoured Myrtaceae pollen while black flying-foxes forage on fruit; although the reverse competitive scenario is a possibility. However, resource partitioning between the two species may be a matter of preference rather than competition, or might differ during times of resource scarcity.

\section{Acknowledgements}

We are extremely grateful to Sue Preston for her assistance in all aspects of field collection.

We would like to thank Peggy Eby for her advice and discussion, Nan Nicholson for her help with seed identification, Jessi Grace and Alex King for provision of fruits to compare to samples and Dr Ruby Chang and Dr Anja Divljan for their discussion of the statistical methods used.

This study was carried out in fulfilment of the Wild Animal Biology MSc degree of PCG at the Royal Veterinary College and the Zoological Society of London. Funding for this research was from the MSc project grant.

\section{Conflict of Interest}

The authors declare no conflict of interest.

\section{References}

Alexander, J. M., Diez, J. M., and Levine, J. M. (2015). Novel competitors shape species' responses to climate change. Nature $\mathbf{5 2 5}, 515-518$.

APSA Members* (2007). 'The Australasian Pollen and Spore Atlas V1.0.' (Australian

National University, Canberra.) Available at http://apsa.anu.edu.au/ 
Ancillotto, L., Santini, L., Ranc, N., Maiorano, L., and Russo, D. (2016). Extraordinary range expansion in a common bat: the potential roles of climate change and urbanisation. The Science of Nature 103, 1-8.

Barclay, M. R., Barclay, L. E., and Jacobs, D. S. (2006). Deliberate insectivory by the fruit bat Rousettus aegyptiacus. Acta Chiropterologica 8, 549-553.

Baldoa, F. and Drake, P. (2002). A multivariate approach to the feeding habits of small fishes in the Guadalquivir Estuary. Journal of Fish Biology 61, 21-32.

Birt, P. (2004). Mutualistic interactions between the nectar-feeding little red flying-fox Pteropus scapulatus (Chiroptera: Pteropodidae) and flowering eucalypts (Myrtaceae): habitat utilisation and pollination. PhD Thesis, University of Queensland, Brisbane.

Birt, P. and Markus, N. (1999). Notes on the temporary displacement of Pteropus alecto and P. poliocephalus by P. scapulatus within a daytime campsite. Australian Mammalogy 21, 107-110.

Chesson, P. (2000). General theory of competitive coexistence in spatially-varying environments. Theoretical population biology 58(3), 211-237.

Eby P. (1996). Interactions between the Grey-headed Flying Fox Pteropus poliocephalus (Chiroptera: Pteropodidae) and its diet plants - seasonal movements and seed dispersal. $\mathrm{PhD}$ Thesis, University of New England, Armidale.

Eby, P., Richards, G., Collins, L., and Parry-Jones, K. (1999). The distribution, abundance and vulnerability to population reduction of a nomadic nectarivore, the Grey-headed Flyingfox Pteropus poliocephalus in New South Wales, during a period of resource concentration. Australian Zoologist 31, 240-253.

Hall, L.S. and Richards, G. (2000). 'Flying Foxes: Fruit and Blossom Bats of Australia.' (UNSW Press: Sydney.) 
Hardin, G. (1960). The competitive exclusion principle. Science 131, 1292-1297.

Hinkle, D. E., Wiersma, W., and Jurs, S. G. (2003). 'Applied statistics for the behavioral sciences.' (5 ${ }^{\text {th }}$ Edition). (Houghton Mifflin: Boston, Massachusetts, USA).

Jones GD (2012). Pollen analyses for pollination research, unacetolyzed pollen. Journal of Pollination Ecology 9, 96-107.

Knudson, C (2018). 'glmm: Generalized Linear Mixed Models via Monte Carlo Likelihood Approximation.' R package version 1.3.0. Available at: https://CRAN.Rproject.org/package $=$ glmm

Lunney, D., Richards, G. and Dickman, C. (2008). 'Pteropus poliocephalus.' The IUCN Red List of Threatened Species 2008: e.T18751A8554062. Available at http://dx.doi.org/10.2305/IUCN.UK.2008.RLTS.T18751A8554062.en [accessed 18 July 2016].

McWilliam, A. N. (1986). The feeding ecology of Pteropus in north-eastern New South Wales, Australia. Myotis 23-24, 201-208.

Nelson, J. E. W. (1965). Behaviour of Australian Pteropodidae (Megachiroptera). Animal Behaviour 13, 544-557.

Oksanen, J., Blanchet, F. G. Kindt, R., Legendre, P., Minchin, P. R., O'Hara, R. B., Simpson, G. L., Solymos, P., Henry, M., Stevens, H. and Wagner, H., (2015). 'vegan: Community Ecology. Package.' R package version 2.2-1. Available at http://CRAN.Rproject.org/package=vegan

Palmer, C., Price, O., and Bach, C. (2000). Foraging ecology of the black flying fox (Pteropus alecto) in the seasonal tropics of the Northern Territory, Australia. Wildlife Research 27, 169-178. 
Palmer, C., and Woinarski, J. C. Z. (1999). Seasonal roosts and foraging movements of the black flying fox (Pteropus alecto) in the Northern Territory: resource tracking in a landscape mosaic. Wildlife Research 26, 823-838.

Parry-Jones, K. (1985). Winter Flying-fox colonies in southern NSW. Australian Zoologist 22(2), 5-6.

Parry-Jones, K. A. (1993). The movements of Pteropus poliocephalus in New South Wales. PhD Thesis, University of New South Wales, Sydney.

Parry-Jones, K. A., and Augee, M. L. (1991). Food selection by Grey-Headed Flying Foxes (Pteropus poliocephalus) occupying a summer colony site near Gosford, New South Wales. Wildlife Research 18, 111-124.

Parry-Jones, K. A., and Augee, M. L. (1992)a. Movements of grey-headed flying foxes (Pteropus poliocephalus) to and from colony site on the central coast of New South Wales. Wildlife Research 19, 331-339.

Parry-Jones, K. A., and Augee, M. L. (1992)b. Insects in flying fox diets. Bat Research News 33, 9-11.

Parry-Jones, K. A., and Augee, M. L. (2001). Factors affecting the occupation of a colony site in Sydney, New South Wales by the Grey-headed Flying-fox Pteropus poliocephalus (Pteropodidae). Austral Ecology 26, 47-55.

R Core Team (2013). 'R: A Language and environment for statistical computing.' $R$ Foundation for Statistical Computing, Vienna, Austria. Available at http://R-project.org/.

Roberts, B. J., Catterall, C. P., Eby, P., and Kanowski, J. (2012). Latitudinal range shifts in Australian flying-foxes: A re-evaluation. Austral Ecology 37, 12-22.

Schmelitschek, E., French, K., and Parry-Jones, K. (2009). Fruit availability and utilisation by grey-headed flying foxes (Pteropodidae: Pteropus poliocephalus) in a human-modified 
environment on the south coast of New South Wales, Australia. Wildlife Research 36, 592600.

Shimeld, P., Hopf, F. and Pearson, S. (2000). Pollen image management: the Newcastle digital collection initiative. Quaternary Australasia 18, 13-15.

Tedman, R.A., and Hall, L.S. (1985). The morphology of the gastrointestinal tract and food transit time in the Fruit Bats Pteropus alecto and P. poliocephalus (Megachiroptera). Australian Journal of Zoology 33, 625-640.

Webb, N. J., and Tidemann, C. R. (1996). Mobility of Australian Flying-Foxes, Pteropus spp.(Megachiroptera): Evidence from Genetic Variation. Proceedings of the Royal Society of London B: Biological Sciences, 263, 497-502.

Welbergen, J. A. (2004). The social organisation of the grey-headed flying-fox, Pteropus poliocephalus. PhD Thesis, University of Cambridge, Cambridge.

\section{Figure and table captions}

Fig. 1. Proportion of faecal samples on a sheet containing certain dietary items as a function of increasing probability (shaded area indicating 95\% confidence intervals) that faecal samples on that sheet are from Pteropus alecto. (a) proportion of faecal samples containing fruit; (b) proportion of faecal samples containing pollen; (c) proportion of dietary items occurring on a sheet which are from an introduced species.

Fig. 2. (a) Relative frequency of occurrence (\%) of fruit (dark grey) and pollen (light grey) in faecal samples, (b) estimated proportions of Pteropus poliocephalus (grey) and P. alecto (black) at four Pteropus colony sites.

Table 1. Description of four flying-fox colony sites in forest fragments within urban areas in north-eastern New South Wales

Table 2. Plant taxa represented in black flying-fox and grey-headed flying-fox faecal samples collected from four colony sites 